\title{
Esophagectomy for benign disease
}

\author{
Jessica Mormando, Arianna Barbetta, Daniela Molena
}

Division of Thoracic Surgery, Department of Surgery, Memorial Sloan Kettering Cancer Center, New York, NY, USA

Contributions: (I) Conception and design: J Mormando, D Molena; (II) Administrative support: None; (III) Provision of study materials or patients: J Mormando, A Barbetta; (IV) Collection and assembly of data: J Mormando; (V) Data analysis and interpretation: All authors; (VI) Manuscript writing: All authors; (VII) Final approval of manuscript: All authors.

Correspondence to: Daniela Molena, MD. Thoracic Surgery Service, Department of Surgery, Memorial Sloan Kettering Cancer Center, 1275 York Avenue, Office: 212.639.3870, New York, NY 10065, USA. Email: molenad@mskcc.org.

\begin{abstract}
Esophagectomy for benign disease is uncommonly used but it is an important option to consider in those patients who have lost function of this organ. Esophageal resection is, in fact considered as a last resort for benign disease, after multiple failed conservative treatments, when the primary disease is not amenable to other treatments and the esophagus has become non-functional leading to very poor quality of life. The indications for esophagectomy for benign diseases can be divided into three major categories: obstruction, perforation and dysmotility. The process leading to organ failure and the need for resection for each specific disease will be discussed in an attempt to provide guidance as to when an esophagectomy is appropriate.
\end{abstract}

Keywords: Esophagectomy; benign esophageal disease; esophageal resection; esophageal stricture; caustic ingestion; gastroesophageal reflux disease (GERD); benign esophageal neoplasm; esophageal perforation; Boerhaave's syndrome; achalasia

Submitted Jan 16, 2018. Accepted for publication Jan 25, 2018.

doi: $10.21037 /$ jtd.2018.01.165

View this article at: http://dx.doi.org/10.21037/jtd.2018.01.165

\section{Introduction}

Esophagectomy for benign disease is generally avoided until all other treatment options have failed. When esophagectomy is considered, the esophagus is usually nonsalvageable and/or quality of life is very poor.

Esophageal resection for benign indications is typically performed for three main functional and/or anatomic problems: obstruction, perforation and/or dysmotility. The most common primary disease processes leading to obstruction are: caustic ingestion, gastroesophageal reflux disease (GERD) and benign neoplasm. Perforation is typically seen as a sequela of Boerhaave's syndrome, iatrogenic injuries, external trauma and caustic ingestion. Dysmotility leading to esophagectomy is usually seen in conjunction with achalasia. However, esophageal spasm and scleroderma can also rarely lead to the need for esophageal resection due to dysmotility.

When performed on appropriate patients, esophagectomy for benign diseases typically have good outcomes and can greatly improve patient's quality of life. The purpose of this article is to describe various indications for esophagectomy in benign disease and provide guidance regarding when an esophagectomy for such conditions should be considered.

\section{Obstruction}

Obstruction from benign esophageal diseases usually occurs due to development of strictures, as is typically the case with caustic ingestion and GERD. However, obstruction can also be caused by neoplasms and complications from previous procedures. Malignant neoplasms are outside of the scope of this paper, however they are the most common indication for esophagectomy. Dilatation and stenting represent the mainstays of treatment for all benign obstructions.

Dilatation has shown to be successful in $75 \%$ of simple strictures after 1-3 treatments (1), and up to $90 \%$ with repeated dilatation (2). Dilatation can be accomplished 


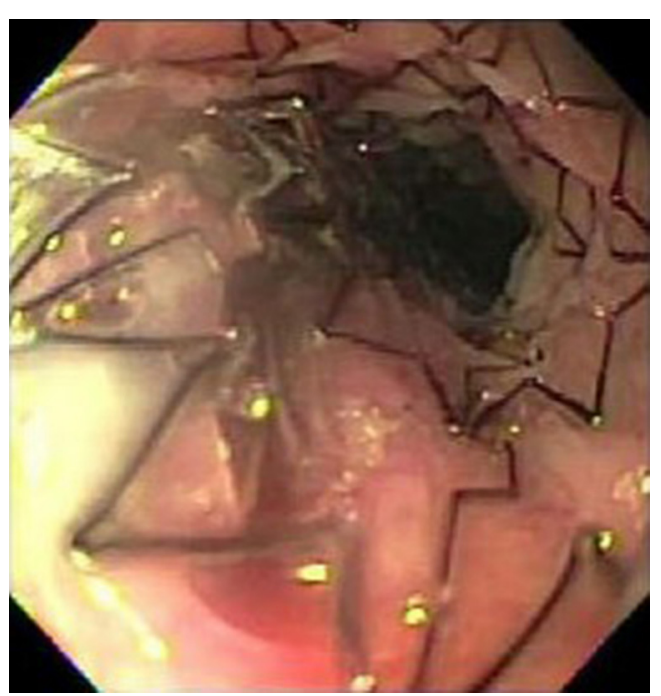

Figure 1 Esophageal stent for benign stricture. The $16 \mathrm{~mm} \times$ $70 \mathrm{~mm}$ ALIMAXX-ES fully covered stent under fluoroscopic guidance. The top of the stent was deployed at $17 \mathrm{~cm}$ and the top of the cricopharyngeal lines at $15 \mathrm{~cm}$.

through either Bougies or balloons. Bougies (either Maloney's or Savary's) are preferred for long fibrotic strictures, while balloons are preferred for short strictures or strictures due to dysfunction. Strictures that are severe or angulated in nature, are longer than $2 \mathrm{~cm}$ or are irregularly shaped are more likely to fail dilatation attempts (2) and depending on severity, may need to be treated with more aggressive measures, such as esophagectomy. For refractory cases, steroids can be injected around the stricture to decrease the inflammatory response in an attempt to prevent stricture re-occurrence. Perforations are the most feared complications of dilatations, and can be especially difficult to treat in these cases due to the decreased healing ability of a severely structured esophagus and the increased luminal pressure above the area of narrowing. The details of perforation management are discussed later, however perforation in this setting, may necessitate an esophageal resection.

Stenting is another viable option for benign esophageal strictures. There are three main types of stents: Metal, plastic and biodegradable (Figure 1). Uncovered metal stents are associated with a high rate of failure. These stents are also associated with ulceration and tissue ingrowth, which can complicate stent removal. Covered metal stents are the preferred treatment for benign esophageal strictures due to their lower complication rate and reduced rate of tissue ingrowth. Plastic stents aren't commonly used due to their high rate of migration and therefore need for reintervention. Biodegradable stents appear to be a promising device, however there are few investigative studies completed thus far.

\section{Caustic ingestion}

Caustic ingestion is one of the benign causes of esophageal strictures in both adults and children. This disease process is more commonly encountered in the pediatric populations due to accidental ingestion. In the adult population, caustic ingestion is most commonly associated with an attempted suicide, however rarely accidental ingestions can occur primarily in the mentally handicapped population. Both acidic and alkalotic fluids can cause stricture, however alkalotic ingestion is more common and usually causes more damage (3). Therefore, surgical intervention is more commonly performed with alkalotic agents, while a higher percentage of acidic injuries can be managed medically (4). The most common complications of caustic ingestion include strictures and perforation, however rarely fistulas may also occur. Due to the nature of injury, the distal esophagus is the most commonly damaged portion, which has important implications when considering an esophagectomy.

The primary treatment modalities include medical management and dilatation for strictures as they develop. In the case of a full thickness perforation however, esophagogastrectomy with esophagostomy is likely necessary, with the goal of delayed reconstruction. Strictures should be dilated as they arise without a delay in treatment. Delaying dilatation after injury leads to refractory strictures and increases the likelihood of an esophagectomy (5).

Esophageal resection in this population is usually considered after the patient has failed multiple serial dilatations. Endoscopic treatment is considered to have failed if there has been no sustained widening of the esophageal diameter after 3-6 months of treatment. Esophagectomy for caustic injury tends to be more common in children due to their resilience to surgery and the need for longer-term treatment (5). Esophageal resection for caustic injury tends to be one of the less complicated surgeries for benign disease. Since the distal esophagus is the most commonly injured segment, the anastomosis is usually less complicated and the esophagus used for the anastomosis has healthy tissue (4). Additionally, since caustic ingestion is primarily an intraluminal disease, it is unlikely 


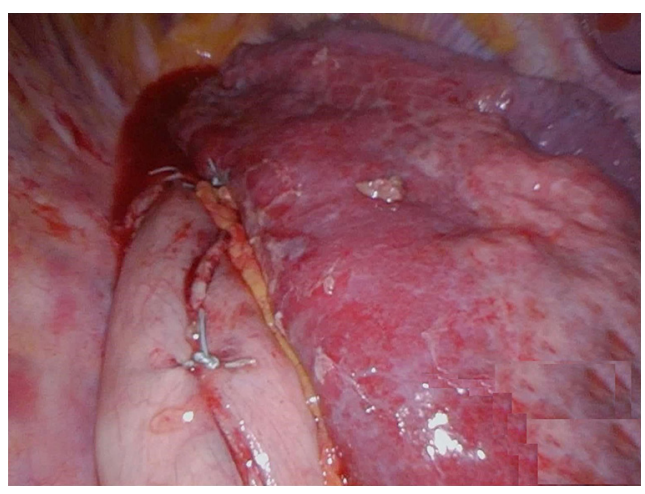

Figure 2 A 5 -cm wide gastric tube created by dividing the stomach with multiple firings of a linear stapler to re-establish continuousness of alimentary tract after distal resection of the esophagus. The gastric conduit is completely mobilized and tubularized to ensure adequate length for the anastomosis.

that these patients have developed an excessive amount of scar tissue around the esophagus that would complicate dissection. Reconstruction of the alimentary tract after esophagectomy for caustic ingestion is done either with gastric pull-up or colonic repositioning. Gastric pull-up is usually the procedure of choice since it is easier to perform and often the conduit length needed is favorable (Figure 2). However, it is important to first ensure that the gastric mucosa was not damaged by the ingested chemical and is healthy enough to serve as the esophageal conduit. It is unusual for damage to the stomach to be severe enough to eliminate the option of a gastric pull-up in these cases but when it happens either colonic or jejunal interposition are preferred (4).

\section{GERD}

Severe GERD when left untreated can lead to peptic stricture. Esophageal strictures are the result of chronic inflammatory damage to the esophageal mucosa. The repeated cycles of esophageal damage and healing leads to scar tissue formation, which eventually causes the development of strictures. Esophageal shortening usually develops when inflammation spreads transmurally to the level of the outer longitudinal layer (6).

Similar to other causes of esophageal stricture, dilation is typically the first line treatment in GERD. Stents are considered for strictures that have failed multiple dilation attempts. Surgical intervention is considered for especially resistant cases. It is important to control the GERD with double dose proton pump inhibitors (PPI) while the stricture is being treated. After successful resolution of the stricture, fundoplication (Nissen or Toupet) should be considered for long-term GERD control (6).

Esophagectomy for GERD is rare and reserved for patients who have developed refractory stricture, severe dysmotility, cancer or complications of previous fundoplication (severe stricture, multiple hernia recurrence, perforations or fistulas). When esophageal resection is performed after one or more failed fundoplication, scar tissue and herniated stomach usually complicate the dissection. Additionally, less stomach is typically available for conduit construction since the fundus has been used for the fundoplication and sometimes the right gastroepiploic has been divided causing the stomach not to be a viable conduit option. The stomach is able to tolerate some degree of ischemia, but careful evaluation of the blood supply is mandatory for successful healing. Innovative technologies that allow microvasculature perfusion assessment with fluorescent dyes are promising and can be used to evaluate whether the stomach does appear to have an unacceptably compromised blood supply and a colonic conduit should be considered. When the stomach is unexpectedly found to have poor perfusion, delayed reconstruction should be entertained. If the perfusion is poor but without significant ischemia, leaving the stomach in the abdomen will allow improvement of the microcirculation and construction of a reliable gastric conduit in a delayed fashion. If the stomach is completely ischemic and needs to be removed during the esophagectomy, delayed reconstruction will allow better preparation for an intestinal conduit. A study performed by Shen et al. showed that patients with anti-reflux surgeries prior to esophagectomy experienced a longer hospital stay, a higher rate of post-operative complications including a higher rate of esophageal leaks, and a higher rate of reoperation (7). Esophageal resection in this population for a benign stricture should be considered with caution and planned appropriately.

\section{Benign neoplasm}

There are multiple benign neoplasms that may necessitate an esophageal resection including esophageal leiomyomatosis, gastrointestinal stromal tumors (GISTs), schwannoma, granular cell tumors, inflammatory pseudotumors, hemangioma, adenoma, papilloma, fibrovascular polyp and extra-esophageal tumors that have invaded or structured the esophagus. The primary treatment modalities include 


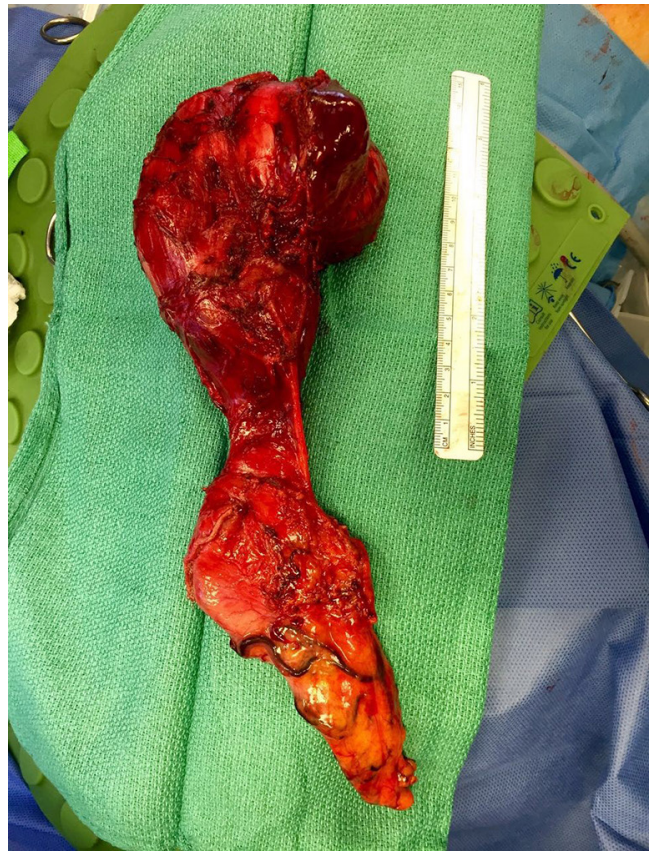

Figure 3 Gross anatomy after esophagectomy for a large and symptomatic gastrointestinal stromal tumor (GIST) of mid esophagus.

dilation, enucleation, endoscopic mucosal resection (EMR) and longitudinal resection with primary repair.

Esophagectomy is considered for benign neoplasm when the lesion causes symptomatic esophageal obstruction, airway obstruction or compression of mediastinal structures that has failed or is not amendable to other treatment modalities. Esophageal resection is also recommended in cases where it is unclear if the lesion is benign or malignant and when disease involves a large or diffuse portion of the esophagus. Leiomyomas are the single most common benign esophageal neoplasm requiring an esophagectomy, which occurs in about $10 \%$ of cases (8). A higher proportion of esophageal resections for leiomyomas are done in children due to the increased risk of multiple tumors and more diffuse esophageal involvement in this age group. Other indications for esophagectomy in leiomyomas include size (generally $8-10 \mathrm{~cm}$ ), annular morphology, multiple or diffuse involvement and concern for leiomyosarcoma (8). Esophageal resection is recommended for GISTs when they become large, however exact size has not been determined. Conservatively, GISTs are taken out when they are larger than $2 \mathrm{~cm}$ (8) (Figure 3). Surgical resection is recommended for schwannomas larger than $2 \mathrm{~cm}$. Schwannomas smaller than $2 \mathrm{~cm}$ can be watched closely, but due to their malignant potential, surgical resection should be considered when feasible. Smaller schwannomas may be removed endoscopically or via enucleation if the lesion is present outside of the mucosa (which they often are). Larger schwannomas require an esophagectomy (8).

\section{Perforation}

The common causes of esophageal perforation include Boerhaave's syndrome, external trauma, caustic ingestion or iatrogenic injury. The esophagus is most likely to perforate at the cricopharyngeus muscle, the bronchoaortic narrowing and the distal esophageal-gastric junction. Iatrogenic perforations most likely occur at Killian's triangle due to the lack of posterior esophageal musculature (9). Esophageal perforation can lead to significant morbidity and mortality if not treated promptly. Biancari et al. found a pooled mortality of $11.9 \%$ for all esophageal perforations. This number was even higher at $13.2 \%$ with intraabdominal perforations. Additionally, a delay in treatment over 24 hours was correlated with a significant increase in mortality by $12.9 \%(10)$.

Perforation can be treated in different ways depending on the location, the extent of the tear and the patient's general conditions. Small tears in the cervical esophagus can heal without intervention, especially if the tear is posterior and anatomically difficult to reach. For larger tears or if there is risk of mediastinal contamination, a cervical incision with or without the use of a drain will be sufficient to control the infection. Most of these will close via granulation by themselves (9). Primary repair is usually the preferred option for thoracic or abdominal esophageal tears if the patient is clinically stable and able to tolerate surgery. Esophageal stents have been recently introduced as a valid alternative to primary repair with promising results, however they must be used with intravenous (IV) antibiotics, mediastinal or pleural drainage, gastric decompression and nutritional support (9). These stents are typically removed 4-6 weeks from placement.

Esophageal resection is rarely used but occasionally necessary for esophageal perforation. Large perforations over $5 \mathrm{~cm}$ with extensive mediastinal or abdominal contamination and pre-existing benign or malignant strictures necessitate urgent esophagectomy. In a retrospective review by Abu-Daff et al., it was shown that $67 \%$ of patients fulfilling these criteria, eventually needed to undergo an esophageal resection (11). Esophagectomy is also the preferred treatment when there has been a delay in 


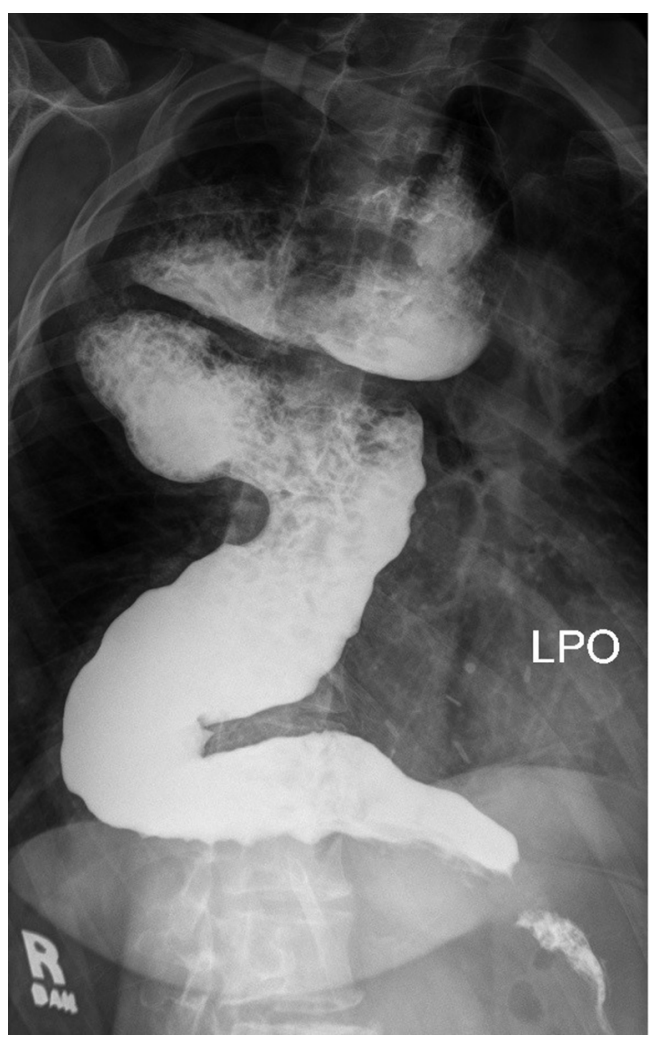

Figure 4 Barium swallow showing end-stage achalasia, with typical bird's beak aspect at the gastroesophageal junction and a large and sigmoid dilatation of the esophagus (megaesophagus). LPO, left posterior oblique.

diagnosis for over 24 hours, due to extensive contamination and sepsis (9). Instrument related perforations rarely benefit from an esophagectomy and can be treated endoscopically in the majority of cases (9). Esophageal resection is also indicated for patients who have failed endoscopic treatment, primary surgical repair or have developed strictures nonresponsive to multiple dilations (9). Iannettoni et al. in a study of 25 patients who underwent primary repair for esophageal perforations, showed that $40 \%$ of patients had to undergo dilation(s) for dysphagia in their post-operative course. Fifty percent of these patients had to eventually undergo an esophagectomy (12). When the patient is too unstable to undergo an esophagectomy, drainage and diversion techniques should be applied and esophagectomy and reconstruction delayed when the patient's conditions have improved.

\section{Dysmotility}

Dysmotility refers to esophageal diseases that interfere with the physiologic movement of the esophagus. The most frequent and better-known disease process under this category is achalasia, however other disorders such as spasm and scleroderma may also cause symptoms significant enough to warrant an esophageal resection. An esophagectomy for dysmotility is indicated, like with other esophageal diseases, when the esophagus is no longer functional and other treatment modalities have failed. End stage esophageal disease is defined in achalasia as a large dilatation of the esophagus with non-responsive reflux, retention of food or presence of pre-neoplastic lesions (13).

\section{Achalasia}

Achalasia is defined as the inability of the lower esophageal sphincter to relax, which in term, causes elevated baseline intraluminal esophageal pressure. This eventually leads to esophageal dilation, which in some cases can be extreme. The cause of achalasia is degeneration or loss of the inhibitory activity of ganglion cells in the myenteric plexus. The lack of the lower esophageal sphincter to relax causes dysphagia and regurgitation of food. The goal of treatment in these patients is to relieve the dysphagia and allow oral nutritional intake.

The gold standard for treatment of symptomatic achalasia is a Heller myotomy with or without a fundoplication for all good surgical candidates. The addition of a fundoplication during the original procedure has gained favor due to the increased risk of GERD after myotomy. A partial wrap (Dor or Toupet) is recommended in order to prevent dysphagia (13). Pre-oral endoscopic myotomy has also been successful in relieving dysphagia, however with high incidence of GERD post operatively (13).

Radiological features of end stage disease in achalasia include tortuous (sigmoid) and massively dilated ( $>6 \mathrm{~cm}$ ) esophagus (Figure 4). Clinically, severe dysphagia, regurgitation, nutritional failure and recurrent episodes of aspiration pneumonia are seen (13). Typically, patients have undergone multiple previous treatments for achalasia, including myotomy. Reasons for failed treatment prior to esophageal resection include an inadequate or healed myotomy, development of GERD and stricture, obstruction from fundoplication wrap, carcinoma or the development of 
a paraesophageal hernia (13).

There has been some disagreement among experts over the role of esophagectomy as primary treatment in patients with a tortuous, sigmoid shaped megaesophagus. Devaney et al. recommended an esophagectomy only in patients with a tortuous, sigmoid esophagus (14). However, a Heller myotomy was recommended for patients with a massively dilated, but straight esophagus (15). Patti et al. showed that laparoscopic myotomy is very successful at reducing dysphagia even when the esophagus is larger than $6 \mathrm{~cm}$ (16). Two additional independent studies showed excellent results using myotomy with a sigmoid esophagus $(17,18)$. Therefore, in our opinion in most patients with a massively dilated and/or sigmoid esophagus, a myotomy should be attempted before an esophagectomy is entertained (13).

Esophagectomy for achalasia is usually more technically challenging than for malignancy due to altered anatomy. Achalasia patients tend to have dense adhesions between the dilated esophagus and the mediastinum due to the chronic inflammatory process of the disease itself and/ or from precious procedures (19). Esophageal deviation is common, usually into the right thoracic cavity and dissection is also complicated by hypertrophied bronchial and aortic vasculature. Achalasia causes hypertrophy of esophageal musculature, which then necessitates vasculature hypertrophy. Mobilization of the esophagus can be further complicated by previous interventions. For these reasons, a transthoracic approach is preferred. Miller et al. showed a higher perioperative blood loss and intraoperative complications associated with a transhiatal approach (20). However, there has been some controversy on this subject. A study conducted by Devaney et al. showed that a transhiatal approach was usually successful and only needed to be converted to a thoracotomy in $6.5 \%$ of patients (14). A minimally invasive approach has been shown to be safe for both a transhiatal and a transthoracic approach by Crema et al. and Schuchert et al. respectively $(21,22)$. Both cervical and thoracic anastomoses are good options, depending on extent of disease and specific patient factors. A vagal sparing esophagectomy can also be considered.

A gastric conduit is the preferred method and most commonly used for achalasia (19). Anastomosis is usually performed in the neck, however, it can be performed in the chest as well. Gastric conduits are preferred due to their good and reliable blood supply and the need for a single anastomosis. Colonic conduits are preferred by some surgeons, however, they are usually used as a second line if the gastric conduit is unsuitable for alimentary tract reconstruction. The advantage of using the colon as a conduit is the avoidance of regurgitation, dumping syndrome and potentially improved anastomotic healing. The disadvantages include impaired peristalsis, food stasis, and poor long-term outcome from elongation and dilation of conduit often requiring revision. Short segment interpositions might avoid some of these problems as shown by Jeyasingham et al. (23). Jejunal conduits have also been used, however, they have been traditionally limited to partial esophageal resections due to limited length of their vascular pedicle. Longer jejunal conduits require supercharging of their vascular pedicles with microvasculature anastomotic techniques (24).

Good results have been reported from numerous series with esophagectomy for achalasia $(14,21,22)$. The majority of patients have excellent symptom control and improved quality of life. However, the risks for esophagectomy are an important consideration when deciding on treatment options. Patient selection is an important determinant of success with esophagectomy for achalasia.

\section{General operative considerations}

Esophagectomy for benign conditions has some important specific aspects to consider. Since there is no need to obtain negative margins, esophagectomy can generally be approached in a less aggressive surgical manner and some anatomic structures that are usually sacrificed in malignant disease can be spared.

Some surgeons have advocated the use of a short segment esophagectomy arguing that the disease is localized and the procedure includes a less complicated dissection (25). We discourage the use of this technique, as it is associated with severe GERD, poor quality of life and potential development of complications (26).

Esophagectomy for benign disease can however require a complex dissection due to the formation of periesophageal adhesions from inflammatory fibrosis, previous surgery and/or previous procedures. Due to the altered anatomy, a transthoracic approach is usually preferred in complicated cases. A transhiatal approach is often used with good outcomes in most resections for benign disease (25). The exact surgical approach should be evaluated and chosen on a case-by-case basis depending on the specific patient, disease etiology and severity.

A vagal sparing technique is something that can be considered for benign esophageal diseases. Vagal nerves are usually compromised in malignant disease due to 
the possibility of transmural spread (25). This technique has shown to preserve gastric acid secretion, gastric emptying, meal capacity and BMI. Dumping syndrome and anastomotic stricture might also be decreased with this approach $(27,28)$. This technique can however lead to anastomotic ulcerations (25).

\section{Conclusions}

Esophagectomy is rarely needed for benign conditions, usually reserved for end-stage disease, when the esophagus is either severely non-functional or when quality of life is very poor. In these circumstances esophagectomy is a good option to consider and it is associated with encouraging success rates and improved quality of life. Depending on the specific disease leading to organ failure, peculiar technical issues should be carefully evaluated in order to avoid complications and optimize results.

\section{Acknowledgements}

None.

\section{Footnote}

Conflicts of Interest: The authors have no conflicts of interest to declare.

\section{References}

1. de Wijkerslooth LR, Vleggaar FP, Siersema PD. Endoscopic management of difficult or recurrent esophageal strictures. Am J Gastroenterol 2011;106:208091; quiz 2092.

2. Carraro EA, Muscarella P. Esophageal replacement for benign disease. Tech Gastrointest Endosc 2015;17:100-6.

3. Zargar SA, Kochhar R, Nagi B, et al. Ingestion of strong corrosive alkalis: spectrum of injury to upper gastrointestinal tract and natural history. Am J Gastroenterol 1992;87:337-41.

4. Harlak A, Yigit T, Coskun K, et al. Surgical treatment of caustic esophageal strictures in adults. Int J Surg 2013;11:164-8.

5. Contini S, Scarpignato C. Caustic injury of the upper gastrointestinal tract: a comprehensive review. World J Gastroenterol 2013;19:3918-30.

6. Barbon C, Mungo B, Molena D, et al. Severe RefluxInduced Esophagitis. In: Pawlik TM, Maithel SK,
Merchant NB. editors. Gastrointestinal Surgery. New York, NY: Springer, 2015:73-85.

7. Shen KR, Harrison-Phipps KM, Cassivi SD, et al. Esophagectomy after anti-reflux surgery. J Thorac Cardiovasc Surg 2010;139:969-75.

8. Ha C, Regan J, Cetindag IB, et al. Benign esophageal tumors. Surg Clin North Am 2015;95:491-514.

9. Cooke DT, Lau CL. Primary Repair of Esophageal Perforation. Oper Tech Thorac Cardiovasc Surg 2008;13:126-37.

10. Biancari F, D'Andrea V, Paone R, et al. Current treatment and outcome of esophageal perforations in adults: systematic review and meta-analysis of 75 studies. World J Surg 2013;37:1051-9.

11. Abu-Daff S, Shamji F, Ivanovic J, et al. Esophagectomy in esophageal perforations: an analysis. Dis Esophagus 2016;29:34-40.

12. Iannettoni MD, Vlessis AA, Whyte RI, et al. Functional outcome after surgical treatment of esophageal perforation. Ann Thorac Surg 1997;64:1606-9; discussion 1609-10.

13. Boeckxstaens GE, Zaninotto G, Richter JE. Achalasia. Lancet 2014;383:83-93.

14. Devaney EJ, Lannettoni MD, Orringer MB, et al. Esophagectomy for achalasia: patient selection and clinical experience. Ann Thorac Surg 2001;72:854-8.

15. Howard JM, Ryan L, Lim KT, et al. Oesophagectomy in the management of end-stage achalasia - case reports and a review of the literature. Int J Surg 2011;9:204-8.

16. Patti MG, Pellegrini CA, Horgan S, et al. Minimally invasive surgery for achalasia: an 8-year experience with 168 patients. Ann Surg 1999;230:587-93; discussion 593-4.

17. Mineo TC, Pompeo E. Long-term outcome of Heller myotomy in achalasic sigmoid esophagus. J Thorac Cardiovasc Surg 2004;128:402-7.

18. Faccani E, Mattioli S, Lugaresi ML, et al. Improving the surgery for sigmoid achalasia: long-term results of a technical detail. Eur J Cardiothorac Surg 2007;32:827-33.

19. Molena D, Yang SC. Surgical management of end-stage achalasia. Semin Thorac Cardiovasc Surg 2012;24:19-26.

20. Miller DL, Allen MS, Trastek VF, et al. Esophageal resection for recurrent achalasia. Ann Thorac Surg 1995;60:922-5; discussion 925-6.

21. Crema E, Ribeiro LB, Sousa RC, et al. Laparoscopic transhiatal esophagectomy for the treatment of advanced megaesophagus. An analysis of 60 cases. Rev Col Bras Cir 2009;36:118-22.

22. Schuchert MJ, Luketich JD, Landreneau RJ, et al. Minimally invasive surgical treatment of sigmoidal 
esophagus in achalasia. J Gastrointest Surg 2009;13:102935; discussion 1035-6.

23. Jeyasingham $K$, Lerut $T$, Belsey RH. Revisional surgery after colon interposition for benign oesophageal disease. Dis Esophagus 1999;12:7-9.

24. Bairdain S, Foker JE, Smithers CJ, et al. Jejunal Interposition after Failed Esophageal Atresia Repair. J Am Coll Surg 2016;222:1001-8.

25. Watson TJ, DeMeester TR, Kauer WK, et al. Esophageal replacement for end-stage benign esophageal disease. J

Thorac Cardiovasc Surg 1998;115:1241-7; discussion

Cite this article as: Mormando J, Barbetta A, Molena D. Esophagectomy for benign disease. J Thorac Dis 2018;10(3):2026-2033. doi: 10.21037/jtd.2018.01.165
1247-9.

26. Watson TJ, Jones CE, Litle VR. Benign diseases of the esophagus. Curr Probl Surg 2009;46:195-259.

27. Banki F, Mason RJ, DeMeester SR, et al. Vagal-Sparing Esophagectomy: A More Physiologic Alternative. Ann Surg 2002;324-35; discussion 335-6.

28. Peyre CG, DeMeester SR, Rizzetto C, et al. Vagal-sparing esophagectomy: the ideal operation for intramucosal adenocarcinoma and barrett with high-grade dysplasia. Ann Surg 2007;246:665-71; discussion 671-4. 